\title{
Detection of topological transitions by transport through molecules and nanodevices
}

\author{
A. A. Aligia ${ }^{1}$, K. Hallberg, ${ }^{1}$ B. Normand, ${ }^{2}$ and A. P. Kampf ${ }^{3}$ \\ ${ }^{1}$ Centro Atómico Bariloche and Instituto Balseiro, \\ Comisión Nacional de Energía Atómica, 8400 Bariloche, Argentina \\ ${ }^{2}$ Département de Physique, Université de Fribourg, CH-1700 Fribourg, Switzerland \\ ${ }^{3}$ Institut für Physik, Theoretische Physik III, Elektronische Korrelationen und Magnetismus, \\ Universität Augsburg, 86135 Augsburg, Germany
}

\begin{abstract}
We analyze the phase transitions of an interacting electronic system weakly coupled to freeelectron leads by considering its zero-bias conductance. This is expressed in terms of two effective impurity models for the cases with and without spin degeneracy. We demonstrate using the halffilled ionic Hubbard ring that the weight of the first conductance peak as a function of external flux or of the difference in gate voltages between even and odd sites allows one to identify the topological charge transition between a correlated insulator and a band insulator.
\end{abstract}

PACS numbers: 73.23.-b, 81.07.Nb, 81.07.Ta, 73.40.Qv

Progress in nanotechnology has made it possible to perform transport experiments on systems as small as single molecules [1]. Metallic 2] or semiconducting [3, 4, 5] quantum dots (QDs) can now be assembled into artificial molecules [4] or solids 2]. QD molecules of different materials and sizes are now being investigated and a wide range of new QD systems is expected to be synthesized in the near future 3, 4]. The transport properties of a finite chain of 15 QDs were first measured over 10 years ago [5] , and the metal-insulator transition has been studied experimentally in a hexagonal lattice of Ag QDs 2]. These advances open the route for new approaches to investigate novel phenomena and theoretical concepts in interacting electron systems.

Here we focus on one such phenomenon, the topological phase transition associated with a parity change of the ground state. The ionic Hubbard model (IHM) with alternating diagonal energy $\pm \frac{1}{2} \Delta$ has received much recent attention [6, 7, 8, 9, 10]. It was proposed to describe the neutral-ionic transition in organic charge-transfer salts [1], and later applied to model ferroelectricity in perovskites [6, 12]. At half filling and in the atomic limit $(t \rightarrow 0)$, the ground state is a band insulator (BI) for $U<\Delta$, but is a Mott insulator (MI) for $U>\Delta$. In one dimension, with non-zero hopping $t$, a spontaneously dimerized insulator (SDI) phase appears between BI and MI. With increasing $U$, one finds first a charge transition at $U=U_{c}$ from BI to SDI, followed by the closing of the spin gap at $U_{s}>U_{c}$, where the transition to the MI occurs. Although in finite systems conventional order parameters such as charge and spin structure factors vary continuously at a phase transition, in a system of $L$ sites one may define charge and spin topological numbers which change discontinuously at $U_{c}(L)$ and $U_{s}(L)$ [8]. The charge Berry phase has a step at $U_{c}(L)$, where a parity change of the ground state occurs for periodic (antiperiodic) boundary conditions if $L=4 m(L=4 m+2$, $m$ integer) [6, 8].

Here we show that this charge transition can be de-

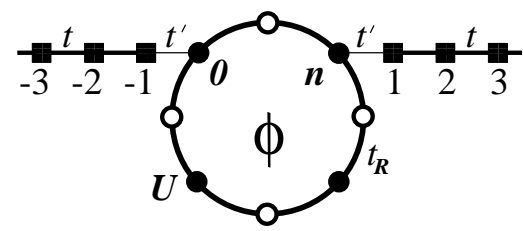

FIG. 1: Interacting electron system on a flux-threaded ring, connected by weak links $\left(t^{\prime}\right)$ to two conducting leads.

tected using the total intensity or width $w$ of the first peak in a zero-bias conductance measurement performed on a flux-threaded ring. The importance of the ring geometry is that the topological transition is absent in an open system [9]. In one of the two parity sectors, which is selected using an applied gate voltage, $w \rightarrow 0$ for applied flux $\phi \rightarrow 0$ if $L=4 m\left(\phi \rightarrow \phi_{0} / 2\right.$, where $\phi_{0}=h c / e$ is the flux quantum, if $L=4 m+2)$. Thus the transition, which is observed by varying parameters such as $\Delta$, may also be studied in ring-shaped molecules where it is not possible to attain a significant threading flux.

The configuration for the proposed experiment is illustrated in Fig. 1. The interacting electron system on the ring is connected to conducting leads through two sites, labeled 0 and $n$, by weak links with hopping $t^{\prime}$, and either two materials or two different gate voltages at alternating sites may be used to model the IHM. It is essential to distinguish between two cases depending on the spin degeneracy of the isolated interacting system for odd particle number. We thus perform two different calculations of the conductance. The first assumes that spin degeneracy is lifted by a Zeeman interaction, but allows orbital degeneracy of the states, which is important when including interference effects arising in a ring geometry [13]. In the second, where spin degeneracy is retained, we map the problem into an effective Anderson model (EAM) and express the conductance as a function of the spectral density of the latter.

If the ground state $|g\rangle$ of the interacting system is non- 
degenerate, for small $t^{\prime}$ the links can be eliminated by a canonical transformation, leading to an effective, noninteracting Hamiltonian for the two leads in which the two "impurity" sites $i$ connected to the links $(-1$ and 1 in Fig. 1) have an energy shift $\Delta \epsilon_{i}(\omega)$, and are connected by an effective hopping $t_{\mathrm{eff}}(\omega)$,

$$
\begin{aligned}
H_{\mathrm{eff}} & =\sum_{k_{L}, \sigma} \epsilon_{k_{L}} c_{k_{L} \sigma}^{\dagger} c_{k_{L} \sigma}+\Delta \epsilon_{-1} c_{-1 \sigma}^{\dagger} c_{-1 \sigma}+\Delta \epsilon_{1} c_{1 \sigma}^{\dagger} c_{1 \sigma} \\
& +\sum_{k_{R}, \sigma} \epsilon_{k_{R}} c_{k_{R} \sigma}^{\dagger} c_{k_{R} \sigma}+\sum_{\sigma}\left(t_{\mathrm{eff}} c_{1 \sigma}^{\dagger} c_{-1 \sigma}+\text { H.c. }\right) .
\end{aligned}
$$

$L(R)$ refers to the lead containing the site $-1(+1)$, and the impurity parameters may be expressed in terms of the Green functions for the isolated ring $g_{i j}(\omega)=\left\langle\left\langle c_{i \sigma} ; c_{j \sigma}^{\dagger}\right\rangle\right\rangle$,

$$
\begin{gathered}
\Delta \epsilon_{-1}(\omega)=t^{\prime 2} g_{00}(\omega), \Delta \epsilon_{1}(\omega)=t^{\prime 2} g_{n n}(\omega), \\
t_{\text {eff }}(\omega)=t^{\prime 2} g_{n 0}(\omega) .
\end{gathered}
$$

The transmittance of $H_{\text {eff }}$ may be calculated in different ways. Without affecting the essential results, we assume identical leads and, for their states without sites $(-1,1)$, we consider two models for the density of states $\rho(\omega)$ and the hybridization $V_{j}(\omega)$ with sites $(-1,1)$. I) $\rho(\omega)$ and $V_{j}(\omega)$ constant: for $j= \pm 1$ and $t_{\text {eff }}=0$, $g_{j j}^{\text {effo }}(\omega)=1 /\left(\omega-\Delta \epsilon_{j}+i \Gamma\right)$ with $\Gamma=\pi \rho V^{2}$. II $)$ Leads described by a tight-binding model with nearest-neighbor hopping $t$, where $g_{j j}^{\text {eff0 }}(\omega)=1 /\left(\omega / 2-\Delta \epsilon_{j}+i \Gamma(\omega)\right)$ with $\Gamma(\omega)=\pi \rho(\omega) V^{2}(\omega)=\sqrt{t^{2}-\omega^{2} / 4}$. By introducing an integer $m=1$ or 2 for cases I and II,

$$
\begin{aligned}
& T\left(\omega, V_{g}\right)= \\
& \frac{4 \Gamma^{2}(\omega)\left|t_{\mathrm{eff}}\right|^{2}}{\left.\left.\left|\left(\frac{\omega}{m}-\Delta \epsilon_{-1}+i \Gamma(\omega)\right)\left(\frac{\omega}{m}-\Delta \epsilon_{1}+i \Gamma(\omega)\right)-\right| t_{\mathrm{eff}}\right|^{2}\right|^{2}} .
\end{aligned}
$$

$V_{g}$ is a gate voltage which changes the on-site energy of all sites of the ring by $-e V_{g}$. In case II this equation is exact $\left(\forall t^{\prime}\right)$ in the non-interacting system, and generalizes a previous result [13]. Eq. (3) also generalizes previous approaches in which only one intermediate state of the ring is included 14, 15], and is appropriate for the study of interference effects 13]. Spin degeneracy limits its validity to magnetic fields $B$ for which the Kondo effect is destroyed, as discussed below. For sufficiently large Zeeman energy $g \mu_{B} B$, the zero-bias conductance at steady state is given by $G=G_{0} T\left(\mu, V_{g}\right) / 2$, where $G_{0}=2 e^{2} / h$ and $\mu$ is the chemical potential of the leads. The results of Ref. [16] suggest that Eq. (3) remains valid, with $G=G_{0} T\left(\mu, V_{g}\right)$, also in the absence of Zeeman splitting in an intermediate temperature range $T_{1}<T<0.1 w$, where $T_{1}$ is very small. Henceforth we set $\mu=0$, corresponding to half-filled leads.

The transmittance as a function of gate voltage is very small except at the poles of $g_{n 0}$. If the ground state $|g\rangle$ has $N$ particles for $\mu=0$, the ground-state energy $E_{g}(N)$ satisfies $E_{g}(N)-e V_{g}<E_{g}(N-1)$ and $E_{g}(N)+$

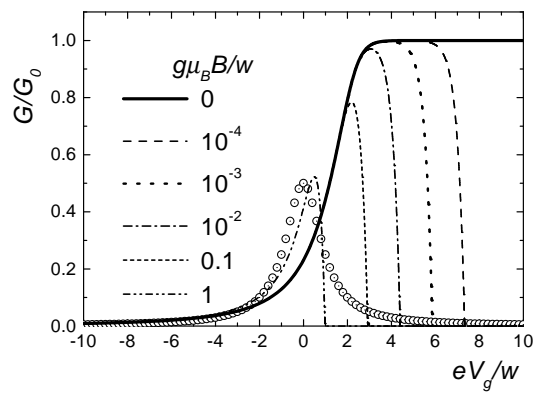

FIG. 2: Conductance as a function of gate voltage for the EAM at several magnetic fields. Circles correspond to the conductance of a resonant level for the majority spin only.

$e V_{g}<E_{g}(N+1)$. As $V_{g}$ is increased, a pole in $g_{n 0}\left(V_{g}\right)$ is reached, and for larger $V_{g}$ the ground state has $N+1$ particles. Similarly, if $V_{g}$ is lowered, a pole is reached when $z=e V_{g}+E_{g}(N-1)-E_{g}(N)=0$.

Assuming that the state of lowest energy for $N-1$ particles, $|g(N-1)\rangle$, is not degenerate, $g_{i j}$ may be expressed as a Laurent expansion around $z=0$,

$$
g_{i j}(z)=\frac{a_{i} \bar{a}_{j}}{z}+\beta_{i j}+\gamma_{i j} z+\ldots,
$$

where $\beta_{j j}, \gamma_{j j}, \ldots$ are real coefficients and

$$
a_{j}=\left\langle g(N-1)\left|c_{j \sigma}\right| g(N)\right\rangle .
$$

$\alpha_{j}=\left|a_{j}\right|^{2}$ is the spectral weight for a local photoemission process. Substituting these expressions in Eqs. (2) and (3), retaining terms to lowest nontrivial order in $t^{\prime} / \Gamma(0)$, and using that $T(z) \sim O(1)$ for $z \lesssim\left(t^{\prime}\right)^{2} / \Gamma(0)$, yields

$$
T(z) \cong \frac{1}{1+(w / z)^{2}} \frac{4 \alpha_{0} \alpha_{n}}{\left(\alpha_{0}+\alpha_{n}\right)^{2}}+O\left(\left(t^{\prime} / \Gamma(0)\right)^{2}\right),
$$

where the half-width at half maximum peak height is

$$
w=\left(\alpha_{0}+\alpha_{n}\right)\left(t^{\prime}\right)^{2} / \Gamma(0) .
$$

Using $G=G_{0} T\left(\mu, V_{g}\right)$, this expression coincides at sufficiently low temperatures with Eq. (7) of Ref. 15.

If sites 0 and $n$ are equivalent by symmetry, then $w=$ $2\left(t^{\prime}\right)^{2} \alpha_{0} / \Gamma(0)$ and the integrated weight $I=\int \mathrm{d} z T(z)=$ $\pi w$ are both proportional to $\left(t^{\prime}\right)^{2}$ and to $\alpha_{0}$. If $V_{g}$ is increased instead of decreased, the same result is obtained with $c_{j \sigma} \rightarrow c_{j \sigma}^{\dagger}$ and $N-1 \rightarrow N+1$ in Eq. (5). Thus a single transport measurement gives simultaneous spectral information related to photoemission and to inverse photoemission. We stress that this information is obtained with much finer energetic resolution $(\mu \mathrm{eV})$ than that available by direct spectroscopic techniques $(\mathrm{meV})$.

We turn next to a calculation of the conductance for small or zero $B$, where either $|g(N)\rangle$ or $|g(N \pm 1)\rangle$ is spin-degenerate. This degeneracy can be taken into account accurately if $w$ is small compared to the separation 
between groups of spin-degenerate energy levels, because the problem becomes equivalent to an EAM. For simplicity we take $V_{g}$ such that a nondegenerate state $|g(N)\rangle$ is close in energy to the spin-degenerate state $|g(N-1)\rangle$, but extension to other cases is straightforward. Neglecting other states, the EAM for any interacting system between the leads is

$$
\begin{aligned}
H_{A}= & -t \sum_{\sigma, i \neq 0,1} c_{i \sigma}^{\dagger} c_{i-1 \sigma}-\sum_{\sigma} d_{\sigma}^{\dagger}\left(t_{-1}^{\prime} c_{-1 \sigma}+t_{1}^{\prime} c_{1 \sigma}\right)+\text { H.c. } \\
& +\varepsilon_{d} \sum_{\sigma} n_{d \sigma}+U_{A} n_{d \uparrow} n_{d \downarrow}-g \mu_{B} B\left(n_{d \uparrow}-n_{d \downarrow}\right), \quad \text { (8) }
\end{aligned}
$$

with $n_{d \sigma}=d_{\sigma}^{\dagger} d_{\sigma}, t_{-1}^{\prime}=t^{\prime} \bar{a}_{0}, t_{1}^{\prime}=t^{\prime} \bar{a}_{n}, \varepsilon_{d}=E_{g}(N)-$ $E_{g}(N-1)-e V_{g}$, and infinite $U_{A}$ (justified because $U_{A}=$ $\left.E_{g}(N)+E_{g}(N-2)-2 E_{g}(N-1) \gg w\right)$.

The conductance is given by

$$
G=\frac{2 e^{2}}{h} \frac{2 \pi w\left|t_{1}^{\prime} t_{-1}^{\prime}\right|^{2}}{\left[\left|t_{1}^{\prime}\right|^{2}+\left|t_{-1}^{\prime}\right|^{2}\right]} \sum_{\sigma} \rho_{d \sigma}(\mu),
$$

where $\rho_{d \sigma}(\omega)$ is the spectral density of the effective $d_{\sigma}$ electrons 17]. The conductance of the EAM computed as a function of gate voltage for several values of $B$ using slave bosons in the mean-field approximation (SBMFA) [19] is shown in Fig. 2. For $B=0, G$ increases abruptly from 0 to $G_{0}$ when $z \sim 0$, and remains nearly perfect for higher $V_{g}$ due to pinning of the Kondo peak in $\rho_{d \sigma}(\omega)$ at the Fermi level. However, because the Kondo energy scale $T_{K}$ decreases exponentially with $V_{g}$, even for very small $B$ the plateau becomes a broad peak, terminated when $T_{K}<g \mu_{B} B$, beyond which $G$ falls to zero. The abruptness of the fall is an artifact of the SBMFA, but the total width of the feature is well described. For larger $B$ the peak width decreases, tending to $w$ to recover the previous result: for $g \mu_{B} B \gtrsim w, G=G_{0} T(z) / 2$, with $T(z)$ given by Eq. (6).

We now apply these results to the topological charge transition in the IHM, which is defined by

$$
\begin{aligned}
H_{\mathrm{IHM}}= & -t_{R} \sum_{i, \sigma}\left(c_{i+1 \sigma}^{\dagger} c_{i \sigma} e^{i \Phi / L}+\text { H.c. }\right)-\frac{1}{2} \Delta \sum_{i}(-1)^{i} n_{i} \\
& -g \mu_{B} B \sum_{i}\left(n_{i \uparrow}-n_{i \downarrow}\right)+U \sum_{i} n_{i \uparrow} n_{i \downarrow},
\end{aligned}
$$

where $n_{i \sigma}=c_{i \sigma}^{\dagger} c_{i \sigma}, n_{i}=\sum_{\sigma} n_{i \sigma}$, and $N=\sum_{i} n_{i}$. We calculate $T\left(V_{g}\right)$ by exact diagonalization for the isolated ring with $L$ sites and $N=L$ electrons, with leads attached to two sites of the same energy $(-\Delta / 2)$ in the presence of a magnetic flux $\phi$ (Fig. 1), with $\Phi=2 \pi \phi / \phi_{0}$. The Green functions $g_{i j}\left(V_{g}\right)$ are obtained numerically and substituted in Eqs. (2) and (3). We choose $L=8$, but similar results are obtained for any $L=4 n(L=$ $4 n+2$ with $\Phi \rightarrow \Phi+\pi)$. The qualitative features are independent of the lead position $n$, with the exception of $n=L / 2$ where the transmittance at $\Phi=\pi$ vanishes for

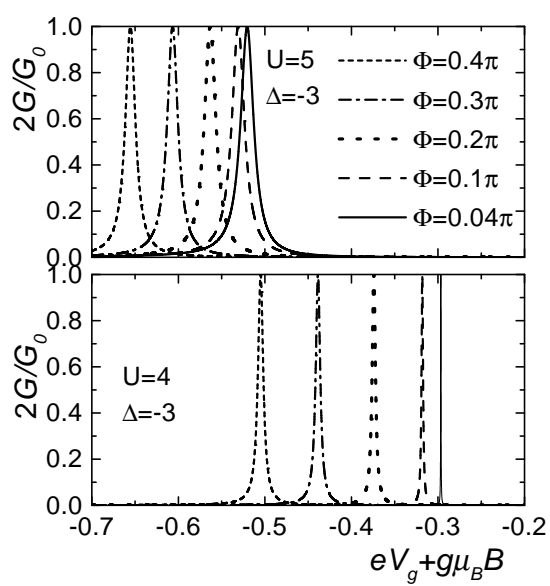

FIG. 3: Transmittance as a function of gate voltage for an 8site IHM ring with leads at sites 0 and 2 for $t_{R}=1, t^{\prime}=0.2$, $\Delta=-3$, and values of $U$ below and above $U_{c}(L=8)=4.352$.

symmetry reasons. Because $H_{\mathrm{IHM}}$ is invariant under simultaneous particle-hole transformation and sign change of $\Delta$, we restrict our analysis to $e V_{g}<0$. We set $t_{R}=1$ as the unit of energy unless otherwise stated.

The topological transition is present at any value of $t_{R}$. As a basis for our study we consider a realistic ring structure containing 8 QDs with only their lowest levels singly occupied, the centers separated by $200 \mathrm{~nm} \mathrm{5]}$, and the parameters $U, \Delta \sim 1 \mathrm{meV}$ and $t_{R} \sim U / 4$ (intermediate coupling strength). We first assume the presence of a strong magnetic field ( $B \sim 1 \mathrm{~T}$ for a $\mathrm{QD}$ array), in the plane of the ring in order not to alter the threading flux, which destroys the Kondo effect. Fig. 3 shows the first peak in $G\left(V_{g}\right)$ at $\Delta=-3$ and for two values of $U$. For $L=8$ and $\Delta= \pm 3$, the topological transition occurs at $U_{c}=4.352$. The peak width is parameter-dependent: for $U=5, w$ is approximately constant with decreasing flux, whereas for $U=4, w$ decreases and the peak disappears at $\Phi=0$. If $U<U_{c}$, the ground state $|g(L)\rangle$ for $\Phi=0$ is even under reflection through any site (corresponding to a BI), while for $U>U_{c}|g(L)\rangle$ is odd (MI or SDI) 8]. For $\Delta=0$, the lowest-energy hole enters the system with the Fermi wave vector, $\pm \pi / 2$, leaving an orbitally degenerate ground state with $L-1$ particles, $|g(L-1)\rangle$. For $\Delta \neq 0$, this degeneracy is broken and $|g(L-1)\rangle$ is odd under reflection through any even site if $\Delta$ is negative [18]. As a consequence, for $U<U_{c}$ the matrix element $a_{0}$ [Eq. (5)] vanishes by symmetry, whence $G$ is negligible at $\Phi=0$. The flux acts as a symmetry-breaking field, which allows one to follow the first peak in a continuous manner until it disappears as $\Phi \rightarrow 0$.

To demonstrate that these essential features are not affected by the presence of a Kondo resonance we consider the 8-site ring with parameters (Fig. 4) similar to one experimental realization [5] and $B$ normal to the ring plane so that the threading flux and Zeeman splitting have the 


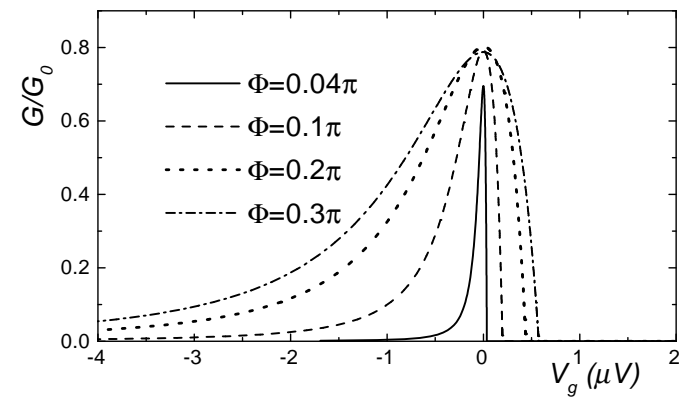

FIG. 4: Transmittance as a function of $V_{g}$ for a ring of 8 QDs described by the IHM. The parameters chosen are $U=1$ $\mathrm{meV}, \Delta=-3 U / 4, t=U / 4, t^{\prime} / t=0.2$, and $g \mu_{B}=0.025$ $\mathrm{meV} / \mathrm{T}$. The curves have been shifted in $V_{g}$ (cf. lower panel Fig. 3) such that their maxima coincide.

same origin. Fig. 4 shows the conductance in this regime, calculated using the EAM in the SBMFA. The disappearance of the feature with decreasing flux remains clear.

The topological transition may now be characterized using $\alpha_{0}$. As shown in Fig. 5 for $\Delta=3, \alpha_{0}$ as a function of $U$ (cf. peak widths in Fig. 3 [18]) vanishes discontinuously at $U_{c}$ for $\Phi=0$, indicating unambiguously the charge transition. The analogous result obtained for fixed $U$ by varying $\Delta$ is of direct experimental interest, because $\Delta$ can be controlled by a difference in gate voltage applied between even and odd sites. A finite flux acts as a parity-breaking perturbation and smooths the transition. This is the situation for artificial arrays of QDs, in which perfect structural symmetry is difficult to attain. We note that curves for all flux values cross approximately at the same point: transport measurements under different applied fields can therefore help to locate the transition, even in the absence of perfect reflection symmetry. However, for a small molecule, which by definition exhibits the $\Phi \rightarrow 0$ limit, the only source of asymmetry is the leads.

In summary, we have considered the transport properties of a ring-shaped interacting system connected weakly to conducting leads. The conductance is expressed in terms of the spectral density of an EAM, which illustrates the breaking of the Kondo effect by an applied field. Outside the Kondo regime we have derived a transmittance formula including nontrivial interference effects. The conductance peaks may be characterized by their total weight, offering spectral information with far higher resolution than conventional spectroscopic measurements. As an application of this result, we have demonstrated that the conductance can be used to detect the charge transition of molecules or quantum dot rings described by the IHM. The method is relevant in the general context of systems presenting phase transitions which involve a symmetry change of the ground state.

A.A.A. and K.H. are fellows of CONICET, and acknowledge the support of the Fundación Antorchas,

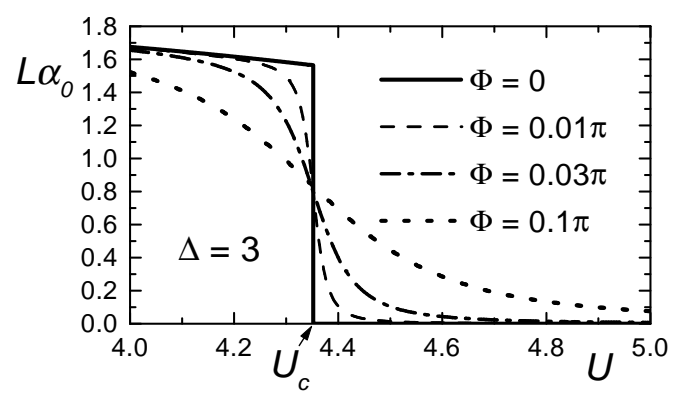

FIG. 5: Matrix element $\alpha_{0}$ as a function of $U$ for $\Delta=3$ $\left(U_{c}=4.352\right)$ over a range of values of the flux $\Phi$.

Project 14116-168, and PICT 03-12742 of ANPCyT. A.P.K. acknowledges support through SFB484 of the Deutsche Forschungsgemeinschaft and B.N. the support of the Swiss National Science Foundation.

[1] M.A. Reed et al., Science 278, 252 (1997); D. Porath et al., Nature (London) 403, 635 (2000); J. Reichert et al., Phys. Rev. Lett. 88, 176804 (2002).

[2] C.P. Collier et al., Science 277, 1978 (1997); G. MedeirosRibeiro et al., Phys. Rev. B 59, 1633 (1999).

[3] A.P. Aliviasatos, Science 271, 933 (1996).

[4] L. P. Kouwenhoven, Science 268, 1440 (1995).

[5] L. P. Kouwenhoven et al., Phys. Rev. Lett. 65, 361 (1990).

[6] R. Resta and S. Sorella, Phys. Rev. Lett. 82, 370 (1999).

[7] M. Fabrizio, A.O. Gogolin, and A.A. Nersesyan, Phys. Rev. Lett. 83, 2014 (1999).

[8] M.E. Torio, A.A. Aligia, and H.A. Ceccatto, Phys. Rev. B 64, 121105 (R) (2001), and references therein. 205109 (2003).

[9] A.P. Kampf et al., J. Phys.: Condens. Matter 15, 5895 (2003); Y.Z. Zhang, C.Q. Wu, and H.Q. Lin, Phys. Rev. B 67, 205109 (2003); C.D. Batista and A.A. Aligia, Phys. Rev. Lett. in press (LJ9218); A. A. Aligia, Phys. Rev. B 69, 041101(R) (2004).

[10] S.R. Manmana et al., cond-mat/0307741 (unpublished).

[11] J. Hubbard and J. B. Torrance, Phys. Rev. Lett. 47, 1750 (1981); N. Nagaosa and J. Takimoto, J. Phys. Soc. Jpn. 55, 2357 (1986).

[12] T. Egami, S. Ishihara, and M. Tachiki, Science 261, 1307 (1994).

[13] E.A. Jagla and C.A. Balseiro, Phys. Rev. Lett. 70, 639 (1993).

[14] G. Chen et al., Phys. Rev. B 50, 8035 (1994).

[15] C.A. Stafford, Phys. Rev. Lett. 77, 2770 (1996).

[16] W. Izumida, O. Sakai, and S. Suzuki, J. Phys. Soc. Jpn. 70, 1045 (2001).

[17] A.A. Aligia and C.R. Proetto, Phys. Rev. B 65, 165305 (2002).

[18] For reflection through odd sites, $|g(L-1)\rangle$ is odd for positive $\Delta$. Under a sign change of $\Delta$ the parities of even and odd sites are interchanged, and with these the charge sector (BI or SDI, MI) in which $\alpha_{0}=0$ at $\Phi=0$.

[19] K. Kang et al., Phys. Rev. B 63, 113304 (2001). 